\title{
The Design and Realization of A New Double Smoothing Filter Algorithm
}

\author{
Fei Niu \\ School of Automation Science and Electrical \\ Engineering \\ Beijing University of Aeronautics and Astronautics \\ Beijing, China \\ Beijing Satellite Navigation Center \\ Beijing, China \\ E-mail: niufei009@sina.com
}

\author{
Yisheng Zhang \\ Beijing Institute of Applied Meteorology \\ Beijing, China \\ E-mail: zhangyisheng118@sina.com
}

\author{
Jianjun Fan \\ Beijing Satellite Navigation Center \\ Beijing, China \\ E-mail: fjjgps@163.com
}

\begin{abstract}
Global Navigation Satellite System is the most important infrastructure to compass the way nowadays and in the future, and the carrier smoothing code method is usually used to improve the accuracy of the position. However, there will be a great influence on satellite navigation and positioning with abnormal ionospheric fluctuations. The filtering divergence problem caused by carrier smoothing code under the abnormal ionospheric conditions is researched in this paper. Then a double smoothing filtering algorithm based on a low pass filer is proposed. This algorithm can improve the positioning accuracy and avoid the filtering divergence through optimizing the smoothing window. Analysis results show that the algorithm proposed by this paper has advantages in two aspects: one is that it can restrain the output noise level of the double smoothing code filter and enhance the detection sensitivity of the abnormal ionosphere fluctuations; on the other hand, the double smoothing code filter can select the optimal smoothing window through the detecting results of the abnormal ionosphere fluctuations, and it can improve the positioning accuracy and avoid the filtering divergence.
\end{abstract}

Keywords-GNSS; SBAS; GBAS; Integrity; Carrier Smoothing Code; Smoothing Filter

\section{INTRODUCTION}

The carrier smoothing code method is selected for aviation navigation terminal to solve the positioning problem generally. The smoothing window time is set as 100 s, under the support of difference information of Satellite Based Augmentation System (SBAS), the horizontal positioning accuracy is better than $1 \sim 2$ meters, the vertical positioning accuracy is better than $2 \sim 5$ meters; under the support of difference information of Ground Based Augmentation System (GBAS), the positioning accuracy can be up to decimeter level[1,2]. The premise of conventional carrier s moothing code algorithm is that the ionosphere state is smooth and the pseudo code and phase have no deviation. However, when there is great fluctuations of ionosphere, the deviation will be produced between pseudo code and phase received by the navigation receiver, and this can lead to filter divergence[3,4]. Aiming at this problem, many domestic and foreign scholars have researched a $\operatorname{lot}[5 \sim 11]$. In this paper, a double smoothing filtering algorithm based on a low pass filer is put forward based on the above literature researches, and the validity of the algorithm is analyzed.

\section{ALGORITHM OF DOUBLE SMOOTHING CODE FILTER}

\section{A. Ionosphere Fluctuation and Its Impact}

The Navigation signal propagation speed is affected by the transmission medium. The delay of code phase and the advance of carrier phase simultaneously happen when the signal passes through the ionosphere. The code phase and the carrier phase are record as:

$$
\begin{gathered}
\rho_{t}=\rho_{\mathrm{t}, \mathrm{IF}}+I_{t}+M_{t, \rho} \\
\phi_{t}=\rho_{\mathrm{t}, \mathrm{IF}}-I_{t}+\lambda N+M_{t, \phi} \\
\rho_{\mathrm{t}, \mathrm{IF}}=r_{t}+c\left[\delta t_{t, u}-\delta t_{t, s}\right]+T_{t}
\end{gathered}
$$

Where $\rho_{\mathrm{t}, \mathrm{IF}}$ is the pseudorange without ionosphere effect; $r_{t}$ is the real range between the receiver and a satellite at time t; $c$ is the light speed; $\delta t_{t, u}$ and $\delta t_{t, s}$ is the clock error of the receiver and the satellite at time $\mathrm{t}$ respectively; $I_{t}$ and $T_{t}$ are the ionospheric and tropospheric delay respectively; $\lambda$ is the wavelength; $N$ is the integer ambiguity; $M_{t, \rho}$ and $M_{t, \phi}$ are the observation error of the pseudorange and carrier phase, including the multipath error.

The pseudorange and carrier phase differences between two adjacent epochs are:

$$
\begin{gathered}
\Delta \rho_{t-1}=\rho_{t}-\rho_{t-1}=\Delta \rho_{\mathrm{t}-1, \mathrm{FF}}+\Delta I_{t-1}+\Delta M_{t-1, \rho} \\
\Delta \phi_{t-1}=\phi_{t}-\phi_{t-1}=\Delta \rho_{\mathrm{t}-1, \mathrm{IF}}-\Delta I_{t-1}+\Delta M_{t-1, \phi}
\end{gathered}
$$


Hatch smoothed the pseudorange at time $\mathrm{t}$ using the recursive filter, written as:

$$
\bar{\rho}_{t}=\frac{1}{k} \rho_{t}+\frac{k-1}{k}\left(\bar{\rho}_{t-1}+\phi_{t}-\phi_{t-1}\right)
$$

where $\mathrm{k}$ is the smoothing window.

According to (6), the weight of the carrier phase is higher than that of the pseudorange in the filter. The ionosphere difference between two observation epochs is not taken into account. During tens of seconds, the ionosphere difference can be usually ignored. However, if ionospheric undulation was severe, the ionospheric delays in pseudorange and carrirer phase would have the same amount of change in the opposite direction. In other words, the deviation between the pseudorange and the carrirer phase is occurred. Thus, the filter will diverges.

\section{B. Algorithm of Double Smoothing Code Filter}

Two smoothing code filters are used in this algorithm, as seen in Fig. 1. The sampling step of the first filter is $k 1$ seconds, the same with the smoothing time of foundation enhanced system. $\mathrm{k} 1$ is $100 \mathrm{~s}$. the sampling step of the first filter is $k 2$ seconds. $k 2$ should be shorter than $k 1$. There are two factors to determine the value of $k 2$. The first is the accuracy of smoothing pseudorange. The second is the sensitivity of filter to the deviation between the pseudorange and the carrirer phase. The difference corrections produced by ground system based on $k l$ smoothing filter are used by the airborne equipment based on $k 1$ smoothing filter; meanwhile, the difference corrections produced by ground system based on $k 2$ smoothing filter are used by the airborne equipment based on $k 2$ smoothing filter.

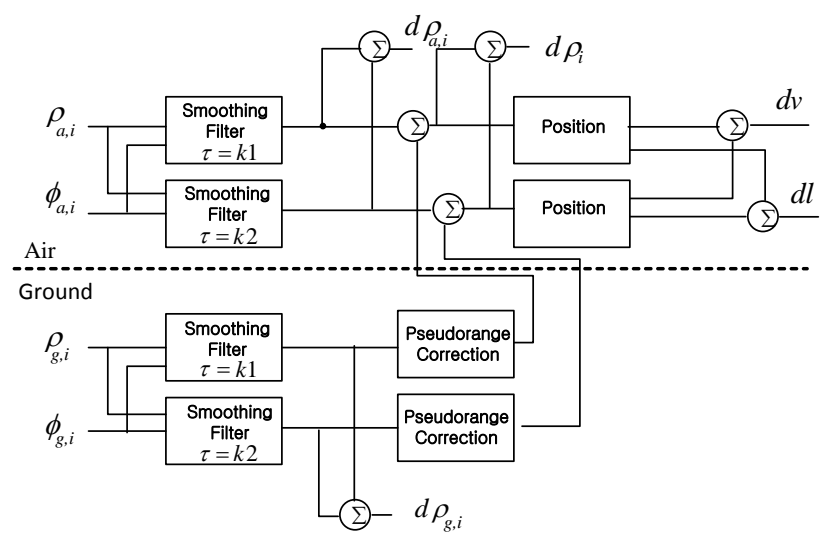

Figure 1. Flow chart of double smoothing code algorithm

The formulas of double smoothing code algorithm are as follows:

$$
\overline{\rho_{a, i, t}^{1}}=\frac{1}{k_{1}} \rho_{a, i, t}+\frac{k_{1}-1}{k_{1}}\left(\bar{\rho}_{a, i, t-1}+\phi_{a, i, t}-\phi_{a, i, t-1}\right)
$$

$$
\begin{gathered}
\overline{\rho_{a, i, t}^{2}}=\frac{1}{k_{2}} \rho_{a, i, t}+\frac{k_{2}-1}{k_{2}}\left(\bar{\rho}_{a, i, t-1}+\phi_{a, i, t}-\phi_{a, i, t-1}\right) \\
d \rho_{a, i}(t)=\overline{\rho_{a, i, t}^{1}}-\overline{\rho_{a, i, t}^{2}}
\end{gathered}
$$

$$
\begin{aligned}
& \overline{\rho_{g, i, t}^{1}}=\frac{1}{k_{1}} \rho_{g, i, t}+\frac{k_{1}-1}{k_{1}}\left(\bar{\rho}_{g, i, t-1}+\phi_{g, i, t}-\phi_{g, i, t-1}\right)(10) \\
& \overline{\rho_{g, i, t}^{2}}=\frac{1}{k_{2}} \rho_{g, i, t}+\frac{k_{2}-1}{k_{2}}\left(\bar{\rho}_{g, i, t-1}+\phi_{g, i, t}-\phi_{g, i, t-1}\right)
\end{aligned}
$$

$$
d \rho_{g, i}(t)=\overline{\rho_{g, i, t}^{1}}-\overline{\rho_{g, i, t}^{2}}
$$

Where $\overline{\rho_{a, i, t}^{1}}$ and $\overline{\rho_{a, i, t}^{2}}$ are the smoothing code of two filters between the airborne receiver and the i-th satellite at time $\mathrm{t} ; \overline{\rho_{g, i, t}^{1}}$ and $\overline{\rho_{g, i, t}^{2}}$ are the smoothing code of two filters between the ground receiver and the i-th satellite; $d \rho_{a, i}(t)$ and $d \rho_{g, i}(t)$ are the double smoothing code of the airborne receiver and ground receiver respectively. The pseudorange abnormality caused by ionosphere storm can be detected and excluded using this algorithm.

\section{Double Smoothing Code Filter Algorithm Based on low-pass filter}

As mentioned above, the double smoothing code algorithm needs a filter with the long smoothing window and the other with short one, then researchers make a subtraction of the two smoothing code and the procession is illustrated as (7) to (12). The shorter time for smoothing window, the more sensitive for the current measurement of pseudorange and quicker the reflection of the ionosphere's abnormal volatility is, but the smoothing result is poorer; on the other hand, the longer time for s moothing window, the better result can be achieved. However, it is not so sensitive for the current measurement of pseudorange and reflection of abnormal fluctuations in ionosphere is also slow. Theoretically, in double smoothing code algorithm, the bigger duration difference of the two smoothing window lengths, and the more sensitive reflection of the ionosphere's abnormity is. However, a negative factor of this design is that noise produced by double smoothing code filter is so large that the abnormal variation of pseudorange domain caused by ionosphere's abnormal fluctuations would be submerged in it. Therefore, it is not useful to test and check the abnormity of the ionosphere. In order to eliminate these adverse factors, this paper puts forward the double smoothing code method on the bas is of low-pass filter processing, and it is shown in Fig. 2. Main principle is that researchers input the output value of the double smoothing code to the low-pass filter, and filter removes high-frequency noise of the pseudorange difference, and then outputs the abnormity in lowfrequency pseudorange. This paper uses the double 
smoothing code filter with the combination of 100 seconds and 10 seconds.

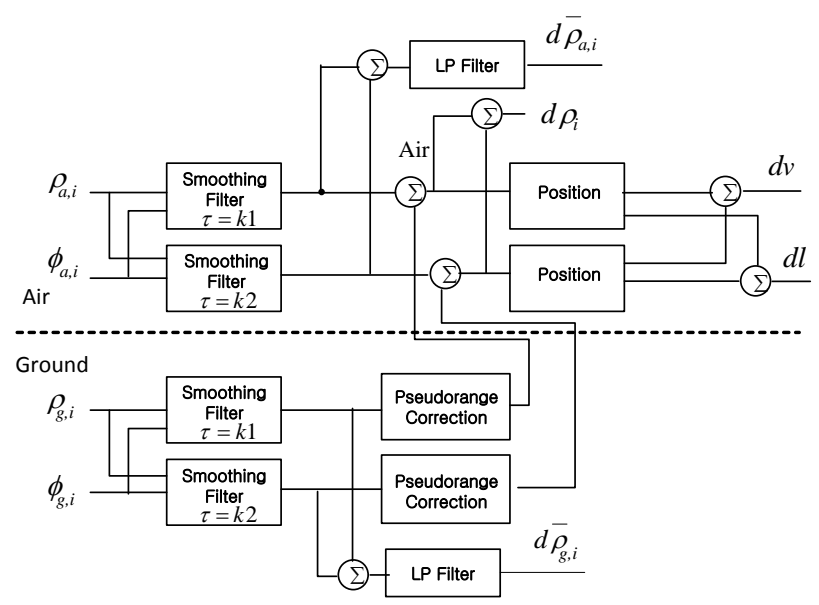

Figure 2. Flow chart of double smoothing code filter algorithm based on low-pass filter

Low-pass filter formula is shown in (13) and (14):

$$
\begin{aligned}
& d \bar{\rho}_{a, i}(t)=(1-k) d \bar{\rho}_{a, i}(t-1)+k d \rho_{a, i}(t) \\
& d \bar{\rho}_{g, i}(t)=(1-k) d \bar{\rho}_{g, i}(t-1)+k d \rho_{g, i}(t)
\end{aligned}
$$

In the formula, $k$ is the weight factor, and in this article researchers set $k=0.01 ; d \bar{\rho}_{a, i}(t)$ and $d \bar{\rho}_{g, i}(t)$ are respectively as double smoothing filter output of ith satellite at time $t$ of airborne receiver and ground system based on low-pass filter.

When the state of ionosphere is smooth and the airborne equipment or ground system have no abnormal fluctuations, researchers use conventional smoothing window of 100 seconds for processing in airborne equipment and ground system; however, if fluctuation occurs in ionosphere, and airborne equipment or ground system detects its abnormity, researchers will use smoothing window of 10 seconds instead.

\section{ANALYSIS OF CASES}

In this paper, MatLab software is selected to simulate for verifying the effectiveness of a low-pass filter based on double smoothing code algorithm and analyzing its impact on the localization performance. GPS constellation simulator conditions are shown in Table I.

TABLE I. GPS ORBITAL PARAMETERS

\begin{tabular}{|c|c|c|l|l|}
\hline $\begin{array}{c}\text { Orbital } \\
\text { plane }\end{array}$ & $\begin{array}{c}\text { Satellites } \\
\text { per orbit } \\
\text { plane }\end{array}$ & $\begin{array}{c}\text { Semi-major } \\
\text { axis }\end{array}$ & Eccentricity & $\begin{array}{c}\text { Orbital } \\
\text { inclination }\end{array}$ \\
\hline 6 & 4 & 26559.80 & 0 & 55 \\
\hline
\end{tabular}

User equivalent range error UERE is synthetic error, each error is independent and obey zero mean Gaussian distribution, and the relating error values are shown in Table II.
TABLE II. CLASSIFICATION ERROR SOURCES

\begin{tabular}{|c|c|}
\hline Error Sources & \\
\hline Satellite component error & 1.0 \\
\hline Ionospheric delay error $/ \boldsymbol{m}$ \\
\hline Tropospheric delay error & 1.6 \\
\hline Receiver noise error & 0.3 \\
\hline Other & 0.3 \\
\hline Sum & 0.5 \\
\hline & 2.0 \\
\hline
\end{tabular}

When the ionosphere fluctuates, the vertical ionospheric delay variation rate is set as $0.008 \mathrm{~m} / \mathrm{s}$. The output of a satellite pseudorange through double smoothing code filter is shown as Fig. 3. As can be seen from the figure, in the first period from 1800 to 3600 seconds, the filter output values has a small amount of change of the step jump, but is overwhelmed by noise substantially.

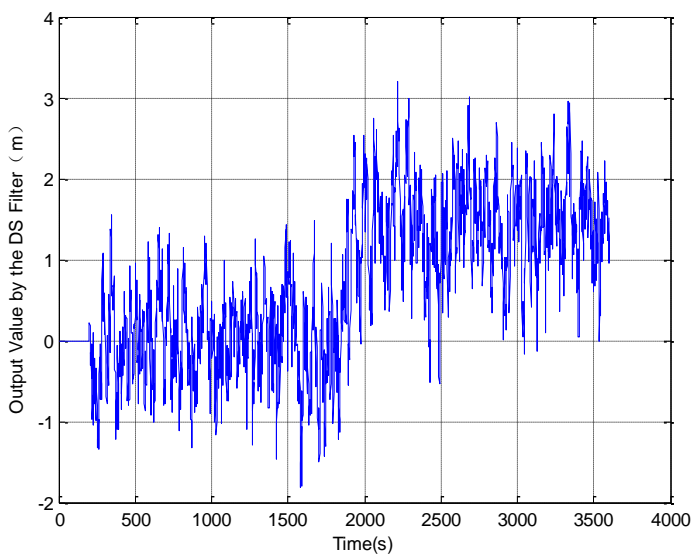

Figure 3. Outputs of double smoothing code filter with abnormal fluctuations ionosphere

Putting the above double smoothing code filter output value into the low-pass filter proposed in this paper, a high-frequency noise can be filtered out and the low frequency component is output, so as to achieve the purpose of detecting abnormal fluctuations in the ionosphere, the result is shown in Fig. 4.

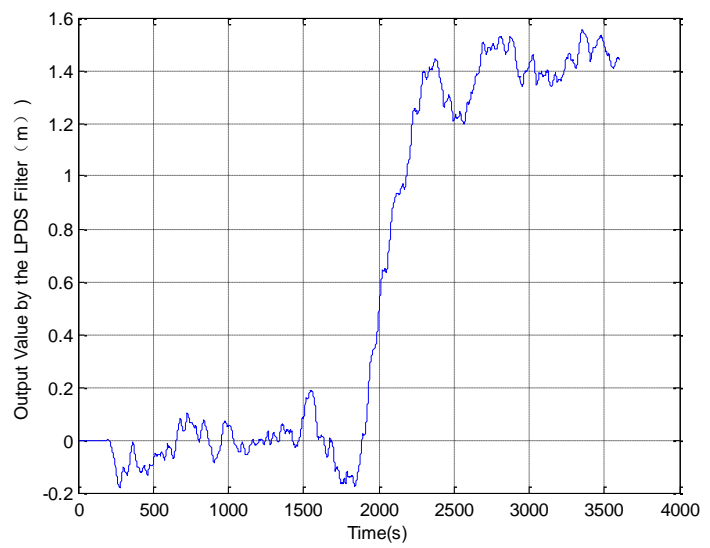

Figure 4. Outputs of double smoothing code filter based on low-pass filter with abnormal fluctuations ionosphere 
Double smoothing code filter algorithm based on a low-pass filter proposed in this paper consis ts of two steps: the first is to determine whether abnormal fluctuations are in the ionosphere by the method described above; then, according to the judgment result, the smoothing window is selected, it is to say that smoothing window with 100 seconds is selected when it is normal, however, smoothing window with 10 seconds is selected when it is abnormal. The impact of double smoothing filter algorithm based on a low-pass filter on positioning performance is analyzed through the following five use cases.

Use Case 1: stable ionosphere, without using carrier smoothing code filter;

Use Case 2: stable ionosphere, using 10 seconds smoothing window;

Use Case 3: stable ionosphere, using 100 seconds smoothing window;

Use Case 4: abnormal fluctuations in the ionosphere, using 100 seconds smoothing window;

Use Case 5: abnormal fluctuations in the ionosphere, using 10 seconds smooth window.
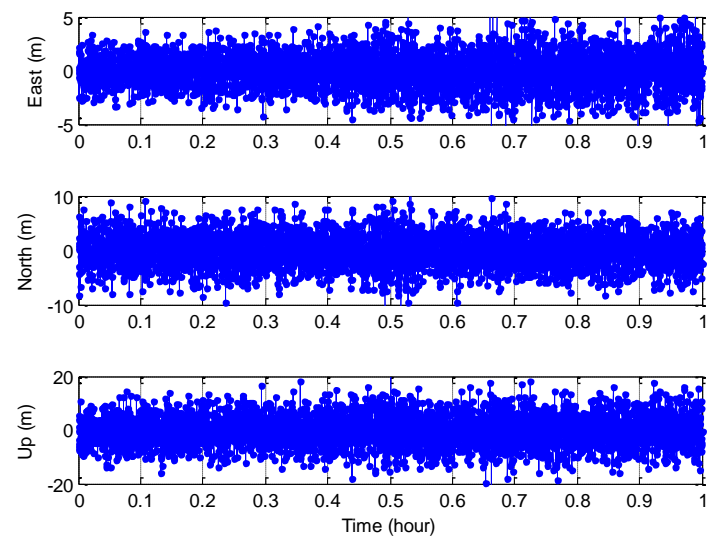

Figure 5. Use Case 1: sequence chart of northeastern positioning error
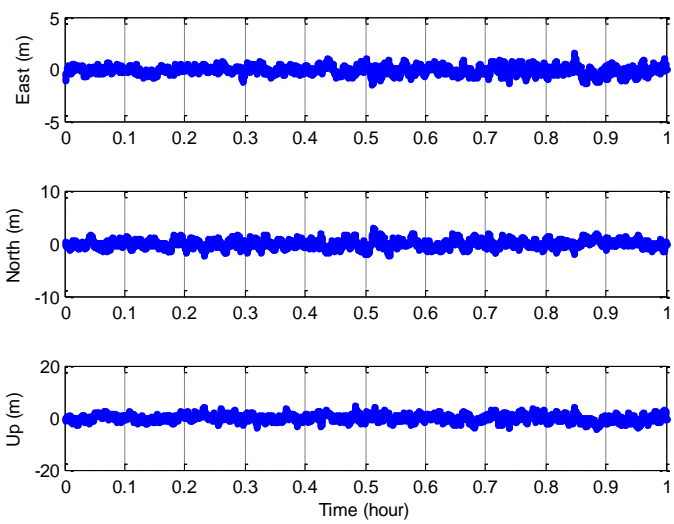

Figure 6. Use Case 2: sequence chart of northeastern positioning error
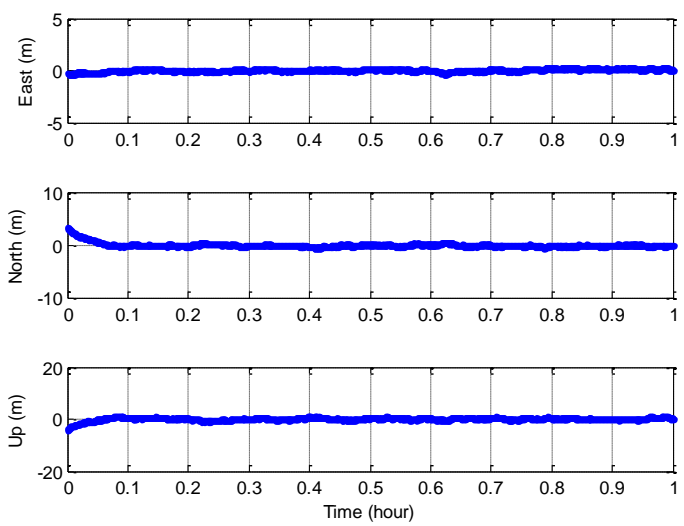

Figure 7. Use Case 3: sequence chart of northeastern positioning error
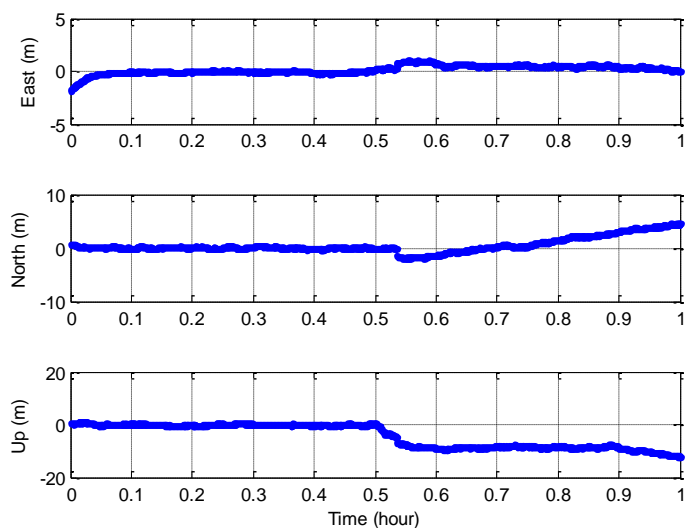

Figure 8. Use Case 4: sequence chart of northeastern positioning error
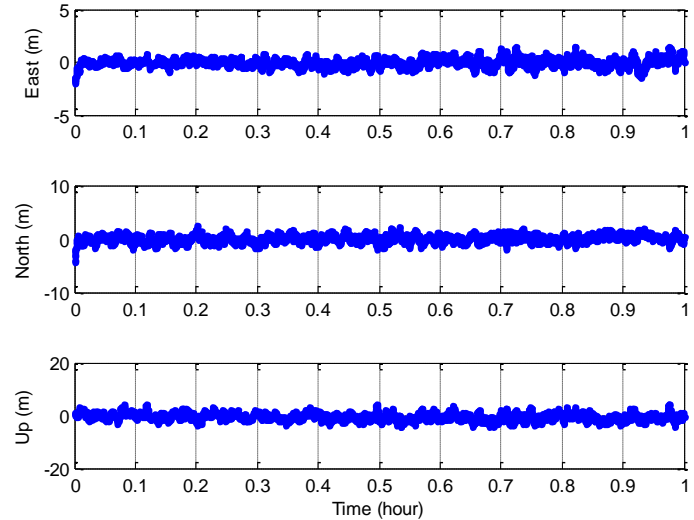

Figure 9. Use Case 5: sequence chart of northeastern positioning error

Statistical results of five cases in northeastern positioning accuracy (Root Mean Square error, RMS) are shown as Table III.

TABLE III. STATISTICAL RESULTS OF FIVE CASES

\begin{tabular}{|l|l|l|l|}
\hline & East (m) & North $(\boldsymbol{m})$ & Up (m) \\
\hline Use Case 1 & 1.83 & 2.91 & 5.91 \\
\hline Use Case 2 & 0.44 & 0.74 & 1.37 \\
\hline Use Case 3 & 0.14 & 0.19 & 0.38 \\
\hline Use Case 4 & 0.55 & 2.14 & 8.65 \\
\hline Use Case 5 & 0.46 & 0.69 & 1.61 \\
\hline
\end{tabular}


Through analysis of the above cases, researchers can see:

(1) Under the condition of stable ionosphere, using a carrier s moothing code method can significantly improve the positioning accuracy, using smoothing window of 100 seconds, the positioning accuracy is better than that of smoothing window with 10 seconds. The basic rule is: the longer time the smoothing window takes, the higher the positioning accuracy will be;

(2) When abnormal fluctuations occur in the ionosphere, the results diverge when using smoothing window with 100 seconds; however, positioning result of smoothing window with 10 seconds is normal, the accuracy is similarly same with that in stable ionosphere. The basic rule is: using a shorter window (10 seconds) can not only improve the positioning accuracy, but also effectively restrain the adverse effect produced by abnormal fluctuations in the ionosphere.

\section{CONCLUSIONS}

All the analysis show that the double smoothing filtering algorithm based on a low pass filer proposed by this paper has advantages in two aspects: one is that it can restrain the output noise level of the double smoothing code filter and enhance the detection sensitivity of the abnormal ionosphere fluctuations; on the other hand, the double smoothing code filter can select the optimal smoothing window through the detecting results of the abnormal ionosphere fluctuations, and it can improve the positioning accuracy and avoid the filtering divergence.

\section{ACKNOWLEDGMENT}

This research was supported by the National Natural Science Foundation of China (41304031, 41374038).

\section{REFERENCES}

[1] RTCA. DO-229D, Minimum Operational Performance Standards for Global Positioning System/Wide Area Augmentation System Airborne Equipment[S]. Washington D.C.:RTCA, 2006.

[2] ICAO. Annex 10 to the Convention on International Civil Aviation, Aeronautical Tele-conmmunications Volume I Radio Navigation Aids[S]. Montreal: ICAO, 1996.

[3] Parkinson. GPS in Global Positioning System Theory and Applications[M]. Washington D.C.: AIAA, 1996.

[4] Walter Todd. The Effects of Large Ionospheric Gradients on Single Frequency Airborne Smoothing Filter for WAAS and LAAS[C]. ION National Technical Meeting, San Diego, 2004:103-109.

[5] Datta Barua, Walter Todd. Using WAAS Ionospheric Data to Estimate LAAS Short Baseline Gradients[C]. ION National Technical Meeting, San Diego, 2002: 523-530.

[6] Yuanxi Yang. Adaptive dynamic navigation and positioning[M]. Beijing: Surveying and mapping press, 2006.

[7] Lin Zhao, Liang Li, Weiquan Huang. An adaptive Kalman filtering algorithm for carrier smoothed code[J]. Journal of Harbin Engineering University, 2010,31(12): 1636-1641.

[8] Fei Niu. Theory and Technique on GNSS Integrity Augment[D]. Zhengzhou: PLA Information Engineering University, 2008.

[9] Gangwang. Research on wide area differential GPS[D]. Zhengzhou: PLA Information Engineering University, 2001.

[10] KONNO H. Design of an aircraft landing system using dualfrequency GNSS[D]. Stanford:Stanford University, 2007.

[11] Suzuki K, Pillen S, Enge P. Evaluation of dual-frequency GBAS performance using data from public receiver networks[C]. ION National Technical Meeting, Savigation, 2010:2592 2602.. 